\title{
Prostaglandin E2 Reverses the Effects of DNA Methyltransferase Inhibitor and TGFB1 on the Conversion of Naive T Cells to iTregs
}

\author{
Mehmet Sahin Emel Sahin \\ Department of Medical Biology, Faculty of Medicine, Gaziantep University, Gaziantep, Turkey
}

\section{Keywords}

Naive T cells · Tregs · TGFB1 · 5-aza-dC · PGE2 - Cytokines

\begin{abstract}
Naturally occurring regulatory T cells (nTregs) are produced under thymic (tTregs) or peripherally induced (pTregs) conditions in vivo. On the other hand, Tregs generated from naive $T$ cells in vitro under some circumstances, such as treatment with transforming growth factor- $\beta$ (TGFB), are called induced Tregs (iTregs). Tregs are especially characterized by FOXP3 expression, which is mainly controlled by DNA methylation. nTregs play important roles in the suppression of immune response and self-tolerance. The prostaglandin E2 (PGE2) pathway was reported to contribute to regulatory functions of tumor-infiltrating nTregs. In this study, we examined whether PGE2 contributes to the formation of iTregs treated with TGFB1 and 5-aza-2'-deoxycytidine (5-aza-dC), which is a DNA methyltransferase inhibitor. We found that the protein and gene expression levels of FOXP3 and IL-10 were increased in 5-aza-dC and TGFB1-treated T cells in vitro. However, the addition of PGE2 to these cells reversed these increments significantly. In CFSE-based cell suppression assays, we demonstrated that PGE2 decreased the suppressive functions of 5-aza-dC and TGFB1-treated T cells.
\end{abstract}

๑) 2019 S. Karger AG, Basel

\section{Introduction}

Naive CD4 ${ }^{+} \mathrm{T}$ cells are continuously produced in the thymus, and they migrate into secondary lymphoid organs, including spleen, lymph nodes, and other lymphoid tissues, where they meet with antigen-MHC complex on the surface of antigen-presenting cells (APCs) [1]. Under certain activation conditions (e.g., in the presence of transforming growth factor- $\beta$ [TGFB]) in vitro, naive $\mathrm{T}$ cells can differentiate into induced regulatory $\mathrm{T}$ cells (iTregs). Unlike iTregs, natural Tregs (nTregs) are generated in vivo and are derived from the thymus or induced in the peripheral circulation and are then called peripherally induced Tregs (pTregs).

Tregs play important roles in many different immune responses [2,3]. Several studies have shown that Tregs are expanded both in tumor microenvironment and peripheral blood of patients with cancer, which is associated with reduced survival and poor prognosis [4]. Alterations in the number and function of Tregs have been implicated in several autoimmune diseases, including multiple sclerosis, active rheumatoid arthritis, and type 1 diabetes. High levels of Tregs have been found in many malignant disorders, including lung, pancreas, and breast cancers, and may also prevent antitumor immune responses, leading to increased mortality.

Tregs, an immune-suppressive subset of $\mathrm{CD}^{+} \mathrm{T}$ cells, are characterized by the expression of the master regulatory transcription factor FOXP3 $[5,6]$. It has been known to be not only the lineage marker for nTregs [6] but also to be expressed transiently in activated $\mathrm{CD} 4^{+}$and $\mathrm{CD} 8^{+}$ $\mathrm{T}$ cells [7]. As a master regulator in the development, differentiation, and immunosuppressive function of Tregs, the expression level of the transcription factor FOXP3 is critical for maintaining immune homeostasis [8]. These regulatory cells can lose FOXP3 expression under certain

Emel Sahin contributed equally to this work.
Prof. Dr. Mehmet Sahin, PhD

E-Mail msahin.sahin44@gmail.com 
conditions, and this is associated with autoimmune pathology [9].

DNA methylation is a common epigenetic mechanism playing important roles in the regulation of gene expression and genomic imprinting for normal development [10]. DNA methyltransferases (DNMTs) are essential components of the methylation process. Any changes in their levels may cause several diseases, such as cancer or autoimmune diseases $[11,12]$. While global hypomethylation increases genomic instability [13] and oncogene production, hypermethylation reduces the production of tumor suppressor genes, which is linked to cancer development $[14,15]$. DNA methylation-modifying drugs have been used for cancer treatment in general. As hypermethylated tumor suppressor genes are considered to be the most common target in cancer, DNMT inhibitors providing hypomethylation of DNA have been thought to be the strong drug candidates [16-18]. Among DNA methylation inhibitors, 5-aza-2'-deoxycytidine (5-aza-dC), a synthetic analogue of cytidine, has been known since the 1960s; however, its approval for cancer treatment has occurred in the 2000s [19]. As a potent antileukemic agent, 5 -aza-dC is highly effective in the treatment of myelodysplastic syndromes $[20,21]$. Changes in DNA methylation status were also reported to have significant effects on the function of $\mathrm{T}$ cells, along with differences in gene expression [12]. The immune system is a promising target for the treatment of both cancer and autoimmune diseases, but it has not been used effectively for therapy for years.

Epigenetic mechanisms, such as histone modification and DNA methylation, are well known to regulate FOXP3 gene expression [22, 23]. Various studies have reported that efficient agents in epigenetic modification, such as 5-aza-dC, can induce FOXP3 expression, promoting the conversion of $\mathrm{CD} 4{ }^{+} \mathrm{CD} 25^{-}$naive $\mathrm{T}$ cells to iTregs because of the promoter demethylation of the FOXP3 gene [24, 25 ] even in preclinical studies [26].

In addition to cancer, the therapeutic potential of demethylating agents for the in vivo treatment of autoimmune diseases has also been explored recently. For example, in mouse models of autoimmune diabetes and colitis, 5-aza- $\mathrm{dC}$ treatment increased Tregs in vivo, decreased autoimmune responses, modulated disease severity, and prolonged survival $[27,28]$. It is well studied that incubation of naive T cells with TGFB and interleukin-2 (IL-2) results in the formation of FOXP $3^{+}$iTregs in vitro $[29,30]$. However, iTregs which were formed by TGFB and IL-2 activation were reported to be unstable compared to 5-aza-dC plus TGFB1 [22]. Another study indicated that 5-aza-dC could induce FOXP3 expression in naive $\mathrm{T}$ cells, but they were not stable for the suppression of responder cells [31].

Prostaglandin E2 (PGE2), synthesized by cyclooxygenase-2 (COX2) enzyme, has multiple effects associated with inflammation and cancer [32]. PGE2 has been reported to contribute to inflammation in experimental disease models [33]. PGE2 has both proinflammatory and anti-inflammatory effects. While PGE2 can suppress the cell function of neutrophils, macrophages, natural killer (NK) cells, T helper (Th) 1 cells, and cytotoxic T cells, it augments the cellular responses of Tregs, Th2, and Th17 cells [34-36]. Additionally, it is known that COX2 expression and the synthesis of PGE2 are associated with cancer progression [37]. PGE2 levels were found to be increased in various cancer types [38, 39]. Recently, there have been several studies reporting that PGE2 supports the regulatory functions of Tregs [32, 40-42]. Despite the COX2/PGE2-dependent induction of Tregs in some studies, there are very few studies showing the effect of PGE2 on naive T cells.

In this study, we investigated the effects of 5-aza-dC with/without TGFB1 on $\mathrm{CD} 4{ }^{+} \mathrm{CD} 25^{-} \mathrm{CD} 45 \mathrm{RA}^{+}$naive $\mathrm{T}$ cells, and we examined to what extent these naive $\mathrm{T}$ cells gained possible Treg functions. We also wanted to examine whether PGE2 synergistically enhances the possible Treg-like properties in 5-aza-dC-treated naive T cells.

\section{Materials and Methods}

\section{Antibodies and Reagents}

The following mouse anti-human antibodies were used for flow cytometry analysis and cell sorting: CD4-PerCP Cy5.5 (BD 560650), CD45RA-PE-Cy7 (BD 560675), CD25-APC (BD 555434), and FOXP3-PE (BD 560852). Fluorochrome-conjugated mouse anti-human isotypes of these antibodies were used as negative controls for surface and intracellular staining.

Anti-human CD3 monoclonal antibody (OKT3 clone) (Functional Grade, eBioscience 16-0037) was used for in vitro activation of naive T cells at a concentration of $1 \mu \mathrm{g} / \mathrm{mL}$. Anti-human CD28 monoclonal antibody (CD28.2) (Functional Grade, eBioscience 16-0289) was used for co-stimulation of the cells in vitro at a concentration of $1 \mu \mathrm{g} / \mathrm{mL}$. Recombinant human IL-2 protein (Merck Millipore IL002, Darmstadt, Germany) was applied to the cells in culture for polyclonal expansion of T cells at a concentration of 300 $\mathrm{U} / \mathrm{mL}$. Recombinant human TGFB1 (Merck Millipore GF111) was prepared as a stock solution of $10 \mu \mathrm{g} / \mathrm{mL}$ in distilled water. The final concentration of TGFB1 used in cell culture media was $3 \mathrm{ng} /$ mL. Stock solution of 5-aza-dC (Sigma A3656) was prepared with DMSO at a concentration of $91 \mathrm{mM}$. The final concentration of 5 -aza-dC used in cell culture media was $10 \mu \mathrm{M}$. PGE2 (Cayman Chemical, MI, USA) was applied to cells at a concentration of 2.8 $\mu \mathrm{M}(1 \mu \mathrm{g} / \mathrm{mL})$ as used in studies by Sinha et al. [43] and Tomić et al. [44].

\section{Isolation of Peripheral Blood Mononuclear Cells and}

Enrichment of $C D 4^{+} T$ Cells

Peripheral blood (20-25 mL) from healthy volunteers was drawn into tubes containing heparin after their informed consent had been obtained in accordance with protocols approved by the local research ethics committee. Peripheral blood mononuclear cells (PBMC) were recovered by using separating solution $(\mathrm{d}=$ $1.077 \mathrm{~g} / \mathrm{mL}$ ) and density gradient centrifugation and then washed with PBS (pH: 7.2-7.3). Before naive T cells were sorted by FACS, $\mathrm{CD}^{+} \mathrm{T}$ cells had been enriched by MACS (magnetic-activated 

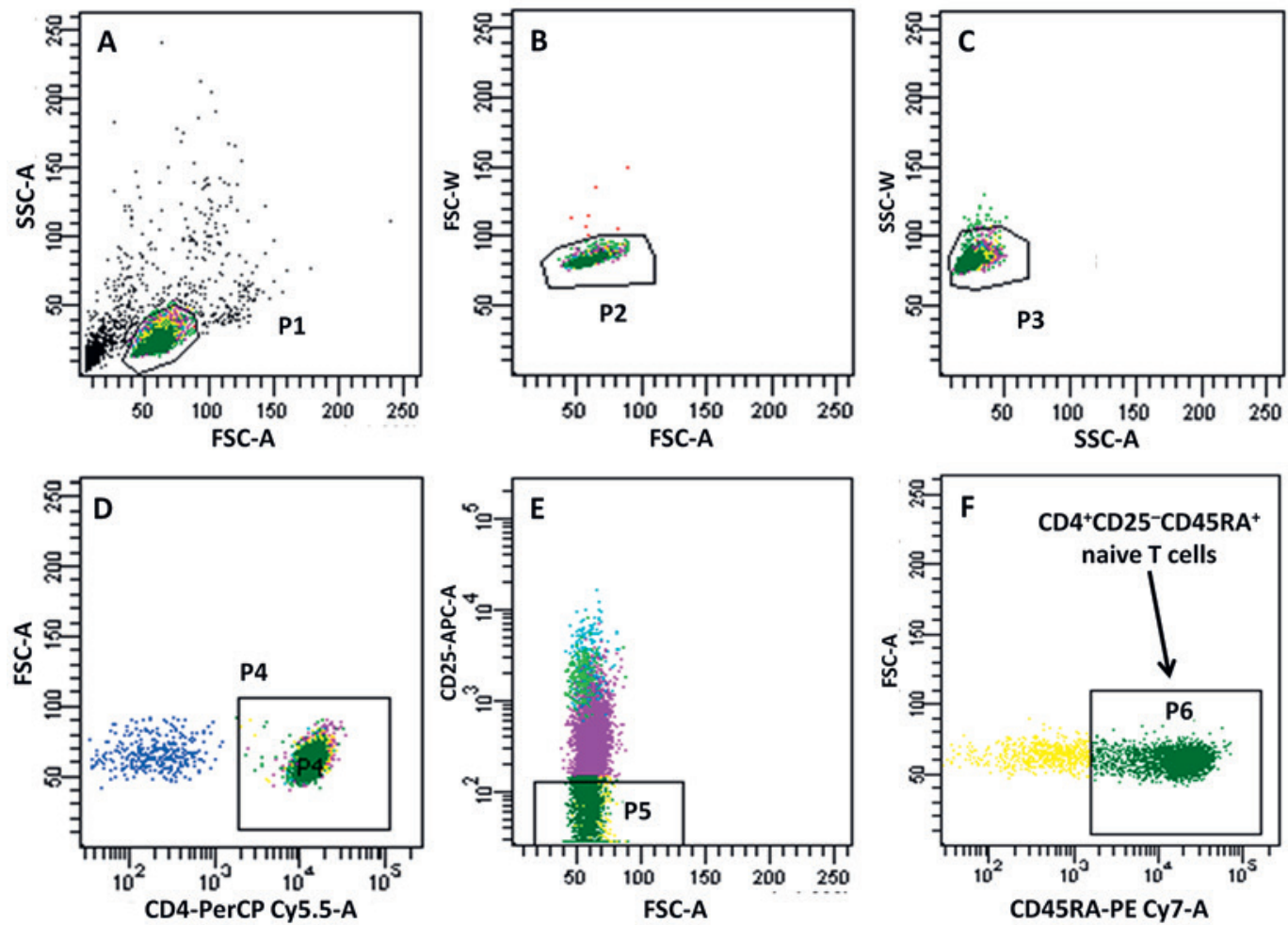

Fig. 1. Flow cytometry gating strategy for sorting of naive T cells. Before the cells were sorted by FACS, CD $4^{+} \mathrm{T}$ cells had been collected by MACS. A Population P1 represents lymphocytes after magnetic sorting of PBMC. B, C Populations P2 and P3 were selected by using FSC-W vs. FSC-A and SSC-W vs. SSC-A dot plots in order to eliminate cell doublets. D The selection of $\mathrm{CD} 4^{+}$cells. E CD25- cells were gated. F Populations in P6 gate represent $\mathrm{CD} 4^{+} \mathrm{CD} 25^{-} \mathrm{CD} 45 \mathrm{RA}^{+}$naive $\mathrm{T}$ cells which were sorted and used for the culture. negative cell sorting) kit (BD IMag, BD Biosciences, CA, USA). In this method, all mononuclear cells are bound with streptavidinconjugated magnetic beads except for $\mathrm{CD}^{+} \mathrm{T}$ lymphocytes. Recovered $\mathrm{CD}^{+}$cells were washed with PBS, centrifuged, and dissolved in PBS.

\section{Cell Surface Staining and Sorting of Naive T Cells}

Following $\mathrm{CD}^{+}{ }^{+}$cell enrichment by using magnetic kit, the cells were centrifuged at $700 \mathrm{~g}$ for $7 \mathrm{~min}$. A cell counting process was performed to determine the amount of antibody conjugated with fluorochrome. The cell surface was stained with anti-CD45RA, anti-CD4, and anti-CD25 for separation and sorting of naive T cells. Titration experiments of the antibodies were performed to calculate the required amount of antibodies. Besides, cells were treated with the isotype control antibodies conjugated with the related fluorochromes in a separate experiment to determine the negative region in flow cytometry chart. The antibodies were incubated with the cells for $30 \mathrm{~min}$ at room temperature. At the end of the incubation, cells were washed twice with cell washing solution $\left(\mathrm{Ca}^{2+}-\mathrm{Mg}^{2+}\right.$-free PBS containing 10\% FBS). After surface staining, $\mathrm{CD} 4{ }^{+} \mathrm{CD} 25^{-} \mathrm{CD} 45 \mathrm{RA}^{+}$naive $\mathrm{T}$ cells were gated and sorted by FACS Aria III Cell Sorter (BD Biosciences) into RPMI 1640 (Invitrogen, CA, USA) containing tubes (Fig. 1). Then, sorted cells were routinely confirmed by flow cytometry and showed about $98 \%$ purity. Cell sorting gates for sub-populations of naive T cells were set as shown in Figure 1.
Expansion of Naive T Cells

Just after the cell sorting process, naive T cells were resuspended in expansion medium and cultured in round bottom 96-well plates at $37^{\circ} \mathrm{C}$ in a humidified $5 \% \mathrm{CO}_{2}$ incubator. Human $\mathrm{AB}$ serum (10\%), $2 \mathrm{~mm}$ glutamine, Na-pyruvate (1\%), non-essential amino acids cocktail (1\%), antibiotic-antimycotic solution (1\%), and HEPES (2\%) solutions were added to RPMI 1640 media, and then its $\mathrm{pH}$ was adjusted to 7.2-7.3. Anti-CD3 (OKT3) and antiCD28 antibodies at a 1:1 ratio were also added into the wells as stimulants. After 2 days, incubated cells were treated with human IL-2 $(300 \mathrm{U} / \mathrm{mL})$. Expansion medium was changed every other day. After 10-12 days of expansion, these activated cells were seeded in appropriate plates and were used for the subsequent experiments.

\section{Intracellular Staining for FOXP3}

Expanded cells were seeded into 96-well petri dishes at an initial density of $1.5 \times 10^{5}$ cells per well. After $24 \mathrm{~h}$, the cells were grouped as control (0.02\% DMSO), 5-aza-dC, 5-aza-dC + TGFB1, PGE2, 5-aza-dC + PGE2, and 5-aza-dC + PGE2 + TGFB1. Reagents and stimulants were administered to the cells at the given doses. At the end of the fourth day, cells were centrifuged at $400 \mathrm{~g}$ with cell washing solution $\left(\mathrm{Ca}^{2+}-\mathrm{Mg}^{2+}\right.$-free PBS containing $10 \%$ FBS), and the supernatants were removed. The cell pellet was resuspended in cell washing solution and transferred into polystyrene round-bottom tubes. Cells were stained with PerCP Cy5.5- 

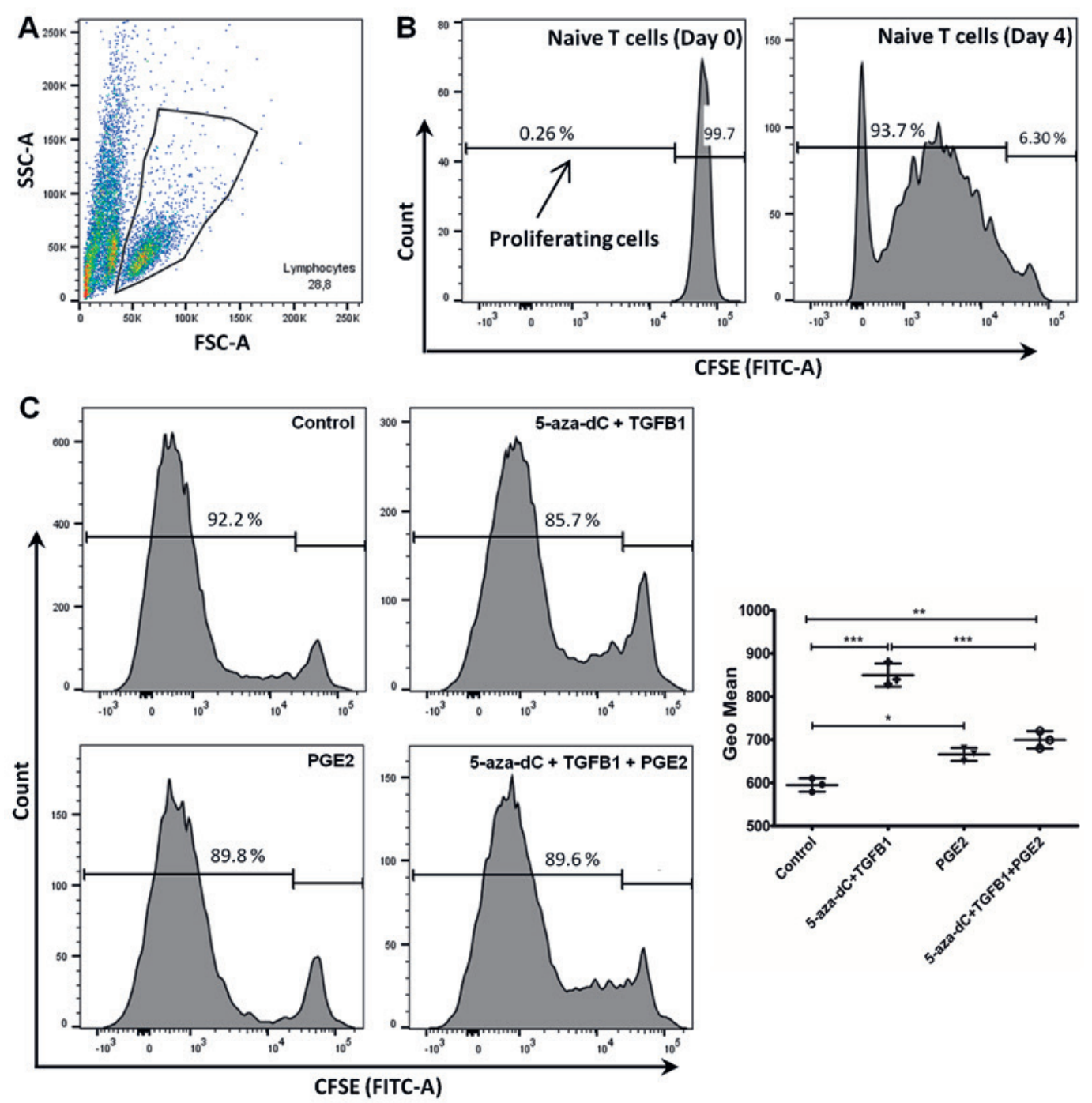

Fig. 2. CFSE suppression assay in co-culture system. A The gated population of lymphocytes. B Only naive T cells, but not co-cultured cells, which were used to determine the proliferating cells. CFSE-stained naive T cells at day 0 (left) and CFSE-stained naive $\mathrm{T}$ cells plus autologous PBMC as feeder cells at day 4, which were stimulated by anti-CD3 and IL-2 $(30 \mathrm{U} / \mathrm{mL})$ (right). C The experimental (co-cultured) groups (control, 5-aza-dC + TGFB1, PGE2, and 5-aza-dC + TGFB1 + PGE2). Activated T cells were pretreated with the 5 -aza-dC $(10 \mu \mathrm{M})+$ TGF- $\beta 1(3 \mathrm{ng} / \mathrm{mL})$ and PGE2 $(2.8 \mu \mathrm{M}$; $1 \mu \mathrm{g} / \mathrm{mL}$ ) reagents alone or in combinations. These pretreated cells

CD4 and APC-CD25 for 30 min at room temperature and then washed with the cell washing solution. A commercially available fixation buffer (BD Biosciences) was used to fix the cell membrane and stabilize the cell, and permeabilization buffer (BD Biosciences) was used to make the cell membrane permeable and facilitate entry of the antibody into the cell. Following the fixation and permeabilization process, cells were stained with PE-FOXP3 antibody and isotype controls for $30 \mathrm{~min}$. After washing, flow cytometric analysis of the cells was performed on FACS Canto II (BD Biosciences) using FACS Diva software (BD Biosciences). were co-cultured with CFSE-stained naive $\mathrm{T}$ cells at a 1:1 ratio. Also, irradiated autologous PBMC were added to this system as a feeder layer. The decreases in the fluorescence intensity of CFSEstained cells represent the cell division as a proliferation indicator on a flow cytometer. While the percentage of the cell population on the right side of the figures indicates the undivided cells, the left region shows the percentage of proliferated cells. The vertical scatter plots show geometric mean fluorescence intensities of proliferating naive T cells \pm SD of triplicate samples. ${ }^{*} p<0.05$; ${ }^{* *} p<0.01$; $* * * p<0.001$.

\section{In vitro Suppression Assay of Activated T Cells Treated with} 5-aza-dC/TGFB1 and PGE2

CFSE dilution by flow cytometry is a method of measuring cellular suppression/proliferation in a co-culture system. CFSE dye binds covalently to cytoplasmic proteins and is diluted as daughter cells divide in course of time. Naive T cells were expanded with anti-CD3/anti-CD28 stimulant antibody at a 1:1 ratio in the presence of IL-2 (300 U/mL) for 10-12 days. Expanded cells were counted and seeded into 96-well petri dishes at a density of $2 \times 10^{5}$ cells. After $24 \mathrm{~h}$, activated T cells were treated with 
the reagents (5-aza-dC, TGFB1, and PGE2) alone and with their combinations. DMSO (0.02\%) was added to the control medium. At the end of the fourth day, the cells in each well were separately collected into different tubes and counted, and then cells were seeded at a density of $1.5 \times 10^{5}$ cells per well into 96 -well plates containing expansion medium. After that, autologous CFSEstained $\mathrm{CD} 4^{+} \mathrm{CD} 25^{-} \mathrm{CD} 45 \mathrm{RA}^{+}$pure naive $\mathrm{T}$ lymphocytes, which were not treated with any reagent, were added into each well at a density of $1.5 \times 10^{5}$ cells to form a co-culture system at a ratio of $1: 1$. In addition, the irradiated autologous PBMC were added into each well at a density of $3.5 \times 10^{5}$ cells. Cells were supplied with anti-CD3 and one-tenth the concentration of IL-2 (30 U/mL). The decrease in the fluorescence intensity of CFSE-stained naive $\mathrm{T}$ cells was analyzed as a proliferation indicator at the end of the fourth day. Cell proliferation was determined by flow cytometric measurement of CFSE dilution on FITC channel. Since only the cells stained with CFSE are pure naive $\mathrm{T}$ cells which were not treated with the reagent, suppressive properties of the drug-supplied activated $T$ cells could be observed. Initial fluorescence intensity of CFSE-stained activated T cells was high as shown in Figure 2B (left). This intensity was reduced during cell division as shown in Figure 2B (right), namely the cell proliferation can be observed by the decrease in fluorescence intensity. Cell suppression assays were carried out by FACS Canto II (BD Biosciences) and analyzed by FlowJo v10 software.

\section{Staining Procedure of Naive T Cells with CFSE}

Expanded $\mathrm{CD} 4{ }^{+} \mathrm{CD} 25^{-} \mathrm{CD} 45 \mathrm{RA}^{+}$pure (not drug-treated) naive $T$ cells were washed with PBS and then counted and resuspended in PBS containing $0.1 \%$ BSA at a density of $3 \times 10^{6}$ cells $/ \mathrm{mL}$. The cells were kept on ice. Stock CFSE solution ( $5 \mathrm{mM}$ ) was diluted to $8 \mu \mathrm{M}$ with PBS containing $0.1 \%$ BSA. While the cell suspension was vortexed, the CFSE solution was pipetted onto it, and thus the final CFSE concentration treated with the cells was adjusted to $4 \mu \mathrm{M}$. Cells were incubated for $10 \mathrm{~min}$ at room temperature. Staining was immediately terminated by adding cold FBS at 3 times as much volume as the vortexed cells, and then cells were allowed to stand on ice for $2 \mathrm{~min}$. Cells were washed 2 times with cell culture medium of $10 \mathrm{~mL}$ and centrifuged at $300 \mathrm{~g}$ for $10 \mathrm{~min}$. Resuspended cells with expansion medium were counted and their fluorescence intensity was confirmed on the FITC channel by flow cytometer.

\section{Enzyme-Linked Immunoassay Assay}

Activated and expanded T cells were seeded into 96-well petri dishes at a density of $1.5 \times 10^{5}$ cells per well. After $24 \mathrm{~h}, 6$ experimental groups were formed as follows: control (0.02\% DMSO); 5-aza-dC; 5-aza-dC + TGFB1; PGE2; 5-aza-dC + PGE2; 5-aza-dC + PGE2 + TGFB1. In these groups, drugs and stimulants were administered at the given doses. At the end of the fourth day, culture media were collected and stored at $-80^{\circ} \mathrm{C}$ until measurement. Secreted IL-10 protein levels in culture medium were assayed by enzyme-linked immunoassay (ELISA) kits (Thermo Fisher Scientific, San Diego, MA, USA), and results were calculated according to the manufacturer's instructions.

\section{Relative Gene Expression Levels by Real-Time PCR}

Activated $\mathrm{T}$ cells were seeded into 24 -well plates at a density of $3.5 \times 10^{5}$ cells per well. After $24 \mathrm{~h}$, reagent cells were administered at the given doses. DMSO (0.02\%) was added to the media of control groups. The cell lysates were homogenized 4 days later by QIAshredder spin columns (Qiagen, MD, USA), and then total RNA was purified using Qiagen RNeasy mini kit (Qiagen) according to the manufacturer's instructions. Total RNA was converted into cDNA by reverse transcription using random hexamers (Applied Biosystems, USA) and $1 \mu \mathrm{L}$ RNase inhibitor $(20 \mathrm{U} / \mu \mathrm{L})$ for each reaction. Obtained cDNAs were stored at $-20^{\circ} \mathrm{C}$ and used for the real-time PCR reactions.

To evaluate the relative mRNA expression levels for the target and reference gene ( $\beta$-actin), real-time PCR was performed using SYBR Green I Master PCR kit (Roche Applied Science, Mannheim, Germany). Light Cycler 480 instrument (Roche Molecular Systems, Inc., CA, USA) and Light Cycler 480SW 1.5 software (Roche Molecular Systems, Inc.) were used for the analysis of gene expressions. We evaluated the results using the method entitled "advanced relative quantification; second derivative max" in the software (note: These results obtained from the software were nearly the same as the results that we calculated by $2^{-\Delta \Delta C t}$ methods). The program for real-time PCR is as follows: heat activation for $10 \mathrm{~min}$ at $95^{\circ} \mathrm{C}$; PCR cycle for $10 \mathrm{~s}$ at $95^{\circ} \mathrm{C}, 15 \mathrm{~s}$ at $57^{\circ} \mathrm{C}, 20 \mathrm{~s}$ at $72^{\circ} \mathrm{C}$ at the total 35 cycles, and single read at the end of each cycle. In addition, we added a melting curve analysis to the real-time PCR program that was done as follows: $5 \mathrm{~s}$ at $95^{\circ} \mathrm{C}, 60 \mathrm{~s}$ at $58^{\circ} \mathrm{C}$, and final target is $97^{\circ} \mathrm{C}$; ramp rate: $0.08 \mathrm{~s}$; read: continuous.

The following primers were used:

- FOXP3: forward: 5'-CATGATCAGCCTCACACCAC-3'; reverse: $5^{\prime}$-CCACTTGCAGACACCATTTG- ${ }^{\prime}$;

- IL-10: forward: 5'-GCCTAACATGCTTCGAGATC-3'; reverse: 5' -TGATGTCTGGGTCTTGGTTC-3';

- IFNG: forward: 5'-GCATCCAAAAGAGTGTGGAG-3'; reverse: $5^{\prime}$ - ATGCTCTTCGACCTCGAAAC-3';

- COX2 (PTGS2): forward: 5'-GTCTGATGATGTATGCCACAATCTG-3'; reverse: $5^{\prime}$ - GATGCCAGTGATAGAGGGTGTTAAA-3';

- $\beta$-Actin: forward: $5^{\prime}$-CATGTACGTTGCTATCCAGGC-3'; reverse: $5^{\prime}$-CTCCTTAATGTCACGCACGAT-3'.

\section{Statistical Analysis}

Comparison of groups according to the parameters was performed using Kruskal-Wallis test (with Bonferroni adjustment) by using Prism from Graph Pad Software (San Diego, CA, USA). $p$ values $<0.05$ were considered statistically significant. Values represent means \pm SD of 3 (flow cytometric and-real time PCR measurements) and 4 (ELISA) independent experiments.

\section{Results}

\section{The Effects of 5-aza-dC, TGFB1, PGE2, and Their Combinations on FOXP3 Protein Levels in Activated T Lymphocytes}

$\mathrm{CD} 4^{+} \mathrm{CD} 25^{-} \mathrm{CD} 45 \mathrm{RA}^{+}$naive $\mathrm{T}$ cells were expanded for 10-12 days under stimulation with anti-CD3, antiCD28, and IL-2 in vitro. The cells were counted and divided into 96-well plates at a density of $1.5 \times 10^{5}$ cells. Cells were treated with the reagents for 4 days and then stained with anti-CD4, anti-CD25, and anti-FOXP3 antibodies. $\mathrm{CD} 25^{+}$cells were gated within the $\mathrm{CD} 4^{+} \mathrm{T}$ cell population.

These activated $\mathrm{T}$ cells stimulated and expanded with anti-CD3, anti-CD28, and IL-2 showed cell surface CD25 and intracellular FOXP3 expression. We observed that $>90 \%$ of lymphocytes differentiated into $\mathrm{CD} 4{ }^{+} \mathrm{CD} 25^{+}$ cells (Fig. 3A). FOXP3 $^{+}$cells in this population were analyzed by flow cytometry (Fig. 3). When exposed to 5-aza-dC alone and co-treatment with TGFB1, these 

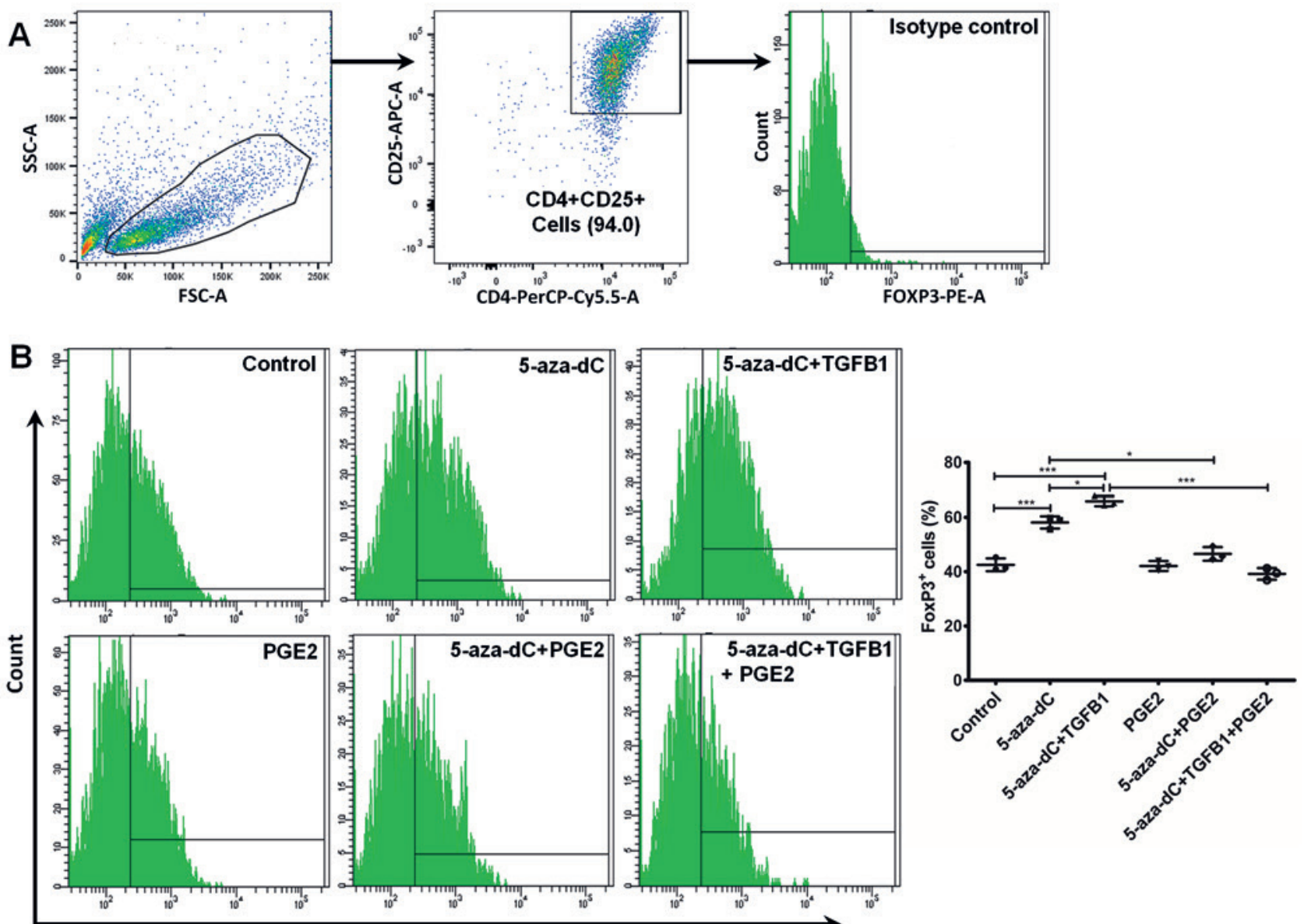

Fig. 3. The effects of 5-aza-dC $(10 \mu \mathrm{M})$, TGFB1 ( $3 \mathrm{ng} / \mathrm{mL})$, PGE2 $(2.8 \mu \mathrm{M} ; 1 \mu \mathrm{g} / \mathrm{mL})$ alone, and co-treatments in combination on FOXP3 expression in activated T cells analyzed by flow cytometry. A Lymphocytes were gated and the $\mathrm{CD} 4^{+} \mathrm{CD} 25^{+}$region was selected in these populations. These gates showed that $\mathrm{CD} 25^{-}$cells were highly converted to $\mathrm{CD} 25^{+}$cells $(>90 \%)$. FOXP3 ${ }^{+}$cells were selected from $\mathrm{CD} 4{ }^{+} \mathrm{CD} 25^{+}$gated cells. Isotype control was used to

activated T cells significantly increased the FOXP3 protein levels compared with the control group. These cells may be considered as iTregs. Although PGE2 alone did not affect the FOXP3 levels, it reversed the enhancing effects of 5 -aza-dC and TGFB1 on FOXP3 expression (Fig. 3B).

5-aza-dC plus TGFB1-Treated Activated T Cells Exhibited Suppressive Properties, but PGE2 Did Not

Demonstrate a Synergistic Effect

We aimed to investigate the effects of the reagents, PGE2, 5-aza-dC, and TGFB1, on suppressive capabilities of activated $\mathrm{T}$ cells. For this aim, the cells exposed to these reagents were co-cultured with pure autologous naive T cells, which were not treated with the drugs, at a 1:1 ratio as mentioned in detail in the Materials and Methods section.

PGE2 Modulates Naive T Cells Treated with 5-aza-dC exclude background signaling. B Flow cytometric illustration showing the proportion of FOXP3 $3^{+}$lymphocytes in experimental groups, including control, 5-aza-dC, 5-aza-dC + TGFB1, PGE2, 5-aza-dC + PGE2, and 5-aza-dC + TGFB1 + PGE2. The vertical scatter plots show the mean percentage of FOXP $3^{+} \mathrm{T}$ cells \pm SD of triplicate samples per group. ${ }^{*} p<0.05 ;{ }^{* * *} p<0.001$.

We observed that 5-aza-dC ( $10 \mu \mathrm{M})$ plus TGFB1 (3 ng/ $\mathrm{mL})$ significantly repressed the proliferation of naive $\mathrm{T}$ cells as compared with the control group (Fig. 2C). PGE2 $(2.8 \mu \mathrm{M} ; 1 \mu \mathrm{g} / \mathrm{mL})$ alone also showed a significant effect on the suppression of CFSE-stained naive T cells compared with the control group. On the other hand, PGE2 reversed the suppressive effects of 5-aza-dC plus TGFB1treated cells on naive T cells (Fig. 2C).

\section{The Relative mRNA Expressions of FOXP3, IL-10,} COX2, and IFNG

Gene expression levels were assayed by real-time PCR method using SYBR Green dye. The relative mRNA expression values of FOXP3, IL-10, COX2, and IFNG were normalized to the $\beta$-actin expression levels as a house- 


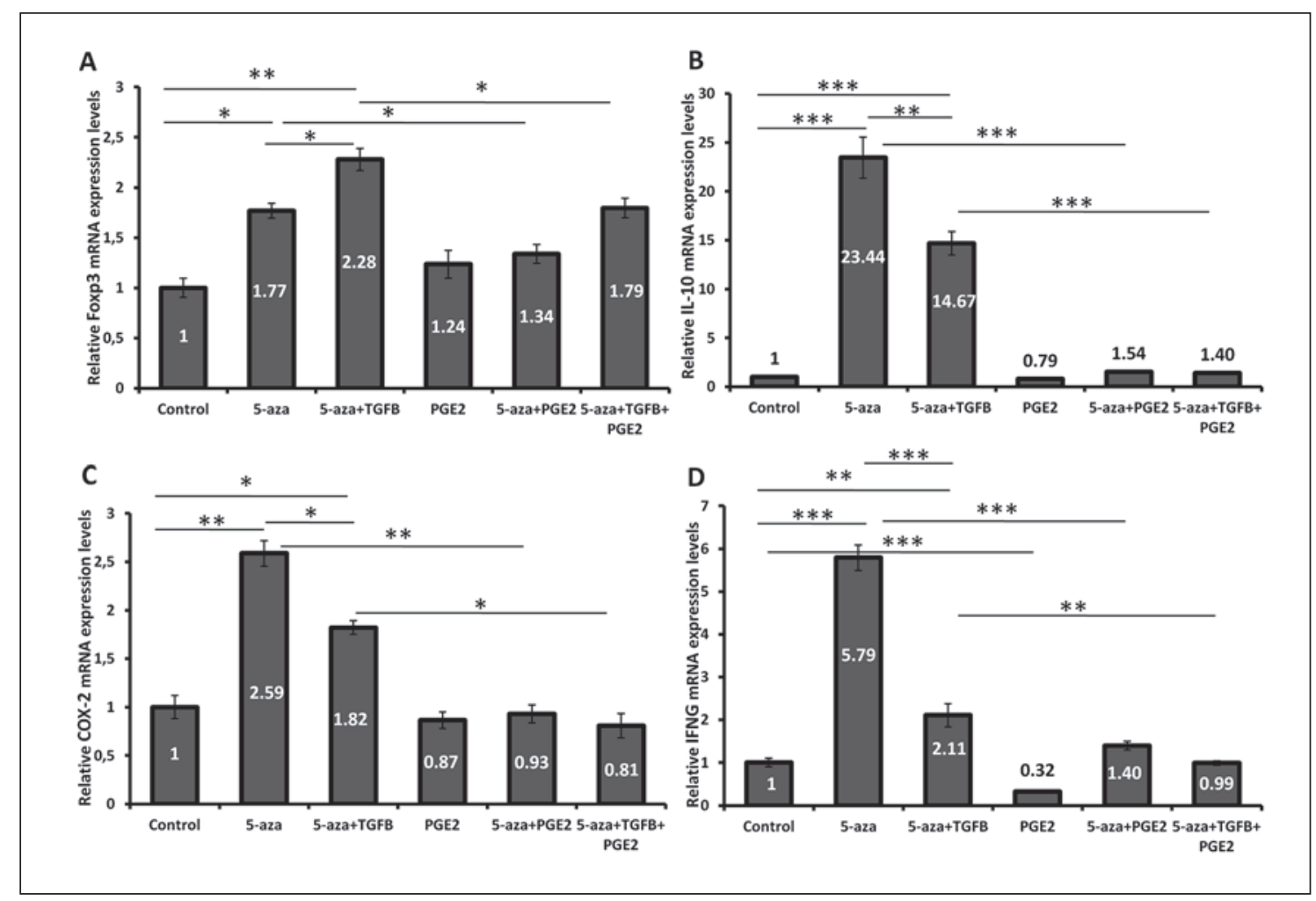

Fig. 4. Effects of 5-aza-dC (10 $\mu \mathrm{M})$, TGFB1 (3 ng/mL), PGE2 (2.8 $\mu \mathrm{M}$ ), and their combinations on FOXP3 (A), IL-10 (B), COX2 (C), and IFNG (D) mRNA expression levels in activated T cells. After detecting the $\mathrm{Cp}$ (crossing point) values of the reference gene and the target gene using the LightCycler ${ }^{\circledR} 480$ SW 1.5 software, the "advanced relative quantification; second derivative max" method was used to determine relative mRNA levels between the groups. Values represent means \pm SD of triplicate samples per group; intergroup significance values were ${ }^{*} p<0.05$; $^{* *} p<0.01$; ${ }^{* * *} p<$ 0.001 .

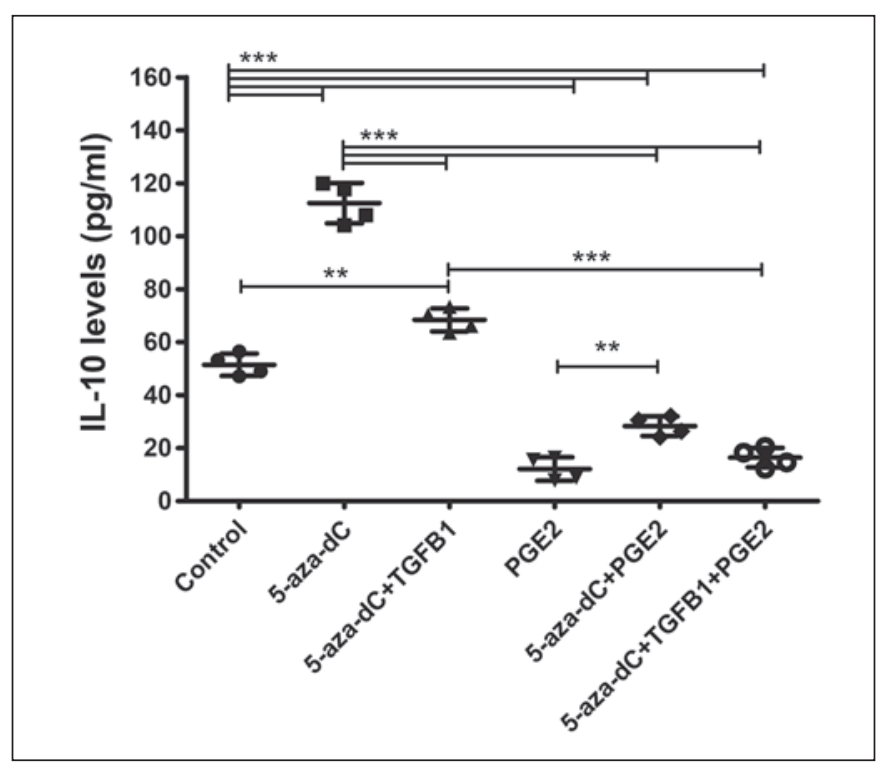

Fig. 5. IL-10 protein levels $(\mathrm{pg} / \mathrm{mL})$ released from activated T cells. The cells were treated with 5-aza-dC $(10 \mu \mathrm{M}), \mathrm{TGFB} 1(3 \mathrm{ng} / \mathrm{mL})$, and PGE2 $(2.8 \mu \mathrm{M})$ alone or in combinations. Secreted protein levels were measured by ELISA according to the manufacturer's protocol in duplicate appropriate dilutions. Values represent means \pm SD. Significance values were expressed as follows: ${ }^{* *} p<0.01$; $^{* * *} p<0.001$. keeping gene. Normalized crossing point $(\mathrm{Cp})$ data were proportioned to the control values (Fig. 4).

5 -aza-dC alone and combined with TGFB1 was found to significantly increase the mRNA level of all the genes as compared to control (Fig. 4A-D). We observed that the changes in the FOXP3 expression were consistent with the results of protein levels obtained from flow cytometry. These increases in expressions by 5 -aza-dC and TGFB1 were observed to be significantly diminished by PGE2 treatment (Fig. 4A-D). It was also seen that PGE2 alone showed about 3 -fold decrease in IFNG expression (Fig. 4D), whereas it did not exhibit any significant effects on other gene expressions (Fig. 4A-C).

\section{IL-10 Protein Levels Released from Activated T Cells}

Naive $\mathrm{T}$ cells cultured and expanded for 10-12 days with anti-CD3, anti-CD28, and IL-2 (100 U) were divided into 6 groups at a density of $1.5 \times 10^{5}$ cells per well as described before. In these groups, released IL-10 levels (pg/ $\mathrm{mL}$ ) in media were measured by ELISA.

The highest increment in IL-10 levels was seen in the 5 -aza-dC group. While 5 -aza-dC in combination with TGFB1 also significantly increased the IL-10 protein, 
PGE2 diminished its levels. Besides, PGE2 substantially reduced the enhancing effects of 5-aza-dC and TGFB1 (Fig. 5).

\section{Discussion}

5-aza-dC exerted its effects as an antineoplastic agent in myelodysplastic syndrome and acute myeloid leukemia patients in clinical trials $[21,45,46]$. The anticarcinogenic effect of 5-aza-dC on solid tumors has also been demonstrated in various preclinical studies $[47,48]$. The therapeutic effects of 5-aza- $\mathrm{dC}$ are mainly explained by 2 mechanisms. The first mechanism is the activation of silenced tumor suppressor genes by demethylation, leading to stopping the progression of the cell cycle, and the second mechanism is the inhibition of cell proliferation by blocking of DNA synthesis $[49,50]$. Although it was demonstrated that 5 -aza-dC treatment increased the number of Tregs through demethylation of the FOXP3 gene in vivo [27], its ability to convert $\mathrm{CD} 4{ }^{+} \mathrm{CD} 25^{-}$naive $\mathrm{T}$ cells into iTregs has been displayed in a limited number of previous studies [24]. TGFB1 is also known to partially induce the promoter and intronic $\mathrm{CpG}$ demethylation in the FOXP3 gene, thereby increasing FOXP3 expression [51].

In this study, we found that 5 -aza-dC with/without TGFB1 significantly increased the percentage of FOXP $3^{+}$ $\mathrm{T}$ cells (Fig. 3B) and the mRNA expression levels of FOXP3 (Fig. 4A) in activated T cells which were stimulated by anti-CD3, anti-CD28, and IL-2 in vitro. We observed that 5 -aza-dC plus TGFB1 was more effective than 5-aza-dC alone in inducing FOXP3 expression (Fig. 3B, $4 \mathrm{~A})$. We also examined whether 5-aza-dC $(10 \mu \mathrm{M})$ plus TGFB1 (3 ng/mL)-treated activated T cells gained the suppressive properties, and, therefore, we performed a CFSE suppression assay. We observed that these agents converted the naive T cells into iTregs (Fig. 2C).

IL-10 is an inhibitory cytokine that mediates the suppressive function of Tregs [52]. IL-10 production was shown to be upregulated by IL-2 in Tregs through the STAT5-responsive intronic enhancer locus [53]. In our study, we found that 5-aza-dC increased the mRNA and protein levels of IL-10 in activated T cells as compared with the control group. Besides, IL-10 was also elevated, but at a lower degree, by the treatment with TGFB + 5-aza-dC (Fig. 4B, 5). Szalmás et al. [54] reported that the promoter of $I L-10$ gene was hypomethylated and transcriptionally active in PBMC, but highly methylated and silent in epithelial cells. In contrast to IL-10, IFNG is a proinflammatory cytokine and it plays essential roles in cancer immune-editing. IFNG, which is secreted by cytotoxic T cells, NK and Th1 cells, promotes the expression of MHC I and II on antigen-presenting cells [55-57]. In addition, this cytokine may suppress the $\mathrm{T}$ cell proliferation. In our study, IFNG may have supported the suppressive effects of iTregs in the 5-aza-dC + TGFB1 group. Wang et al. [58] reported that IFNG treatment could induce the conversion of $\mathrm{CD} 4^{+} \mathrm{CD} 25^{-} \mathrm{T}$ cells into $\mathrm{CD} 4^{+}$ Tregs and enhance the FOXP3 expression and regulatory functions in vitro in human and mouse systems. Our results that were obtained from the co-culture experiments and gene expression assay of IFNG are consistent with this study. We observed that 5-aza-dC (with or without TGFB) increased the relative mRNA expression levels of IFNG as compared to control. Yano et al. [59] showed that IFNG gene could be controlled by promoter methylation. It was also shown that 5 -aza-dC caused promoter hypomethylation and enabled CREB recruitment, and thus IFNG was synthesized in Th2 cells. Although 5-aza$\mathrm{dC}$ increased the relative mRNA expressions of FOXP3, $I L-10, I F N G$, and COX2, TGFB1 was found to modulate the effects of 5-aza-dC in all expressions except for FOXP3 (Fig. 4).

PGE2 is synthesized by COX enzymes and displays its effects via the receptors EP1-4 [60]. This pathway also mediates the regulatory functions of Tregs in tumor microenvironment $[41,42]$. It is known that PGE2 shows different immune responses depending on the cell types, namely it supports Th2 but inhibits Th1 responses such as the production of IFNG [61]. In the present report, we also observed that PGE2 decreased IFNG mRNA expressions about 3 times in activated T cells (Fig. 4). Recently, in another study, we found that PGE2 increased intracellular IL-10 and FOXP3 levels in nTregs $\left(\mathrm{CD} 4^{+} \mathrm{CD} 25^{\mathrm{hi}}\right.$ $\mathrm{CD} 127^{\mathrm{lo}} \mathrm{CD} 4 \mathrm{RA}^{+}$) which were sorted and polyclonally expanded [62]. Baratelli et al. [41] also showed that PGE2 induced FOXP3 gene expression in $\mathrm{CD}^{+} \mathrm{T}$ cells. However, in this study, we did not find any significant effect of PGE2 $(2.8 \mu \mathrm{M})$ on the protein levels of FOXP3 (Fig. 3B), but it slightly increased the mRNA levels (Fig. 4A). Besides, PGE2 decreased the secreted levels of IL-10 protein about 4 times (Fig. 5).

Since the PGE2 pathway is known to play a role in nTreg functions, at first we had thought that the combination of PGE2 with 5-aza-dC/TGFB1 may exert a synergistic effect on activated T cells. However, we did not observe such an effect. In activated $\mathrm{T}$ cells, we observed that PGE2 reversed the enhancing effects of 5-aza-dC/TGFB1 on IL-10, IFNG, FOXP3, and COX2 expression. Also, in the CFSE suppression assay, we found a similar effect of PGE2, but it decreased the proliferated cells when it was used alone as compared to control (Fig. 2C).

In conclusion, the increased expressions of FOXP3, IL10 , and COX2 levels and the decreased number of proliferated cells by 5 -aza-dC/TGFB1 treatment support the idea that the agents may have converted the naive $\mathrm{T}$ cells activated by IL-2, anti-CD3, and anti-CD28 into iTregs. 
In this study, we also concluded that TGFB1 only shows a synergistic effect with 5 -aza-dC on the increase of FOXP3 mRNA and protein levels. Besides, when used together with 5-aza-dC/TGFB1, PGE2 reversed the effects of these agents, namely it did not exhibit a synergistic effect with 5 -aza-dC/TGFB1 in activated $\mathrm{T}$ cells.

We suggest that the reversal effects of PGE2 on iTregs treated with 5 -aza-dC/TGFB1 may be explained by its possible epigenetic modulating properties as indicated by a few studies. Xia et al. [63] showed that PGE2 increased DNA methylation of certain tumor suppressor and DNA repair genes and promoted tumor growth in colorectal cancer cells. Huang et al. [64] also reported that PGE2 induced DNMT and increased global DNA methylation in lung fibroblasts.

These studies are consistent with our findings on the effects of PGE2 on iTregs and can provide a meaningful explanation. Our study is the first to suggest that the inducing effect of PGE2 on DNMT expression may have reversed the hypomethylating capacity of 5-aza-dC. However, an additional study is needed to clarify the possible mechanisms.

\section{Acknowledgements}

We would like to thank Akdeniz University for laboratory facilities. We would also like to thank Prof. Dr. Sadi Köksoy for his laboratory facility.

\section{Statement of Ethics}

We state that subjects have given their informed consent and that the study protocol has been approved by the local research ethics committee on human research.

\section{Disclosure Statement}

The authors declare that they have no conflicts of interest.

\section{Funding Sources}

Funding for this study was provided by grants from TUBITAK (The Scientific and Technological Research Council of Turkey; project number: 112T473).

\section{References}

1 Luckheeram RV, Zhou R, Verma AD, Xia B. CD4囚T cells: differentiation and functions. Clin Dev Immunol. 2012;2012:925135.

2 Sakaguchi S, Powrie F. Emerging challenges in regulatory $\mathrm{T}$ cell function and biology. Science. 2007 Aug;317(5838):627-9.

3 Wang HY, Wang RF. Regulatory T cells and cancer. Curr Opin Immunol. 2007 Apr;19(2): 217-23.

4 Griffiths RW, Elkord E, Gilham DE, Ramani V, Clarke N, Stern PL, et al. Frequency of regulatory $\mathrm{T}$ cells in renal cell carcinoma patients and investigation of correlation with survival. Cancer Immunol Immunother. 2007 Nov; 56(11):1743-53.

5 Takeuchi Y, Nishikawa H. Roles of regulatory $\mathrm{T}$ cells in cancer immunity. Int Immunol. 2016 Aug;28(8):401-9.

6 Hori S, Nomura T, Sakaguchi S. Control of regulatory $\mathrm{T}$ cell development by the transcription factor Foxp3. Science. 2003 Feb; 299(5609):1057-61.

7 Whiteside TL, Schuler P, Schilling B. Induced and natural regulatory $\mathrm{T}$ cells in human cancer. Expert Opin Biol Ther. 2012 Oct;12(10): 1383-97.

8 Sakaguchi S, Miyara M, Costantino CM, Hafler DA. FOXP3+ regulatory T cells in the human immune system. Nat Rev Immunol. 2010 Jul;10(7):490-500.

9 Li X, Zheng Y. Regulatory T cell identity: formation and maintenance. Trends Immunol. 2015 Jun;36(6):344-53.

10 Robertson KD. DNA methylation and human disease. Nat Rev Genet. 2005 Aug;6(8):597610.
11 Jiang L, Gonda TA, Gamble MV, Salas M, Seshan V, Tu S, et al. Global hypomethylation of genomic DNA in cancer-associated myofibroblasts. Cancer Res. 2008 Dec;68(23):99008.

12 Richardson B. DNA methylation and autoimmune disease. Clin Immunol. 2003 Oct; 109(1):72-9.

13 Kulis M, Esteller M. DNA methylation and cancer. Adv Genet. 2010;70:27-56.

14 Joyce BT, Gao T, Zheng Y, Liu L, Zhang W, Dai $Q$, et al. Prospective changes in global DNA methylation and cancer incidence and mortality. Br J Cancer. 2016 Aug;115(4):46572.

15 Hansen KD, Timp W, Bravo HC, Sabunciyan S, Langmead B, McDonald OG, et al. Increased methylation variation in epigenetic domains across cancer types. Nat Genet. 2011 Jun;43(8):768-75.

16 Stresemann C, Brueckner B, Musch T, Stopper H, Lyko F. Functional diversity of DNA methyltransferase inhibitors in human cancer cell lines. Cancer Res. 2006 Mar;66(5):2794800.

17 Esteller M. Relevance of DNA methylation in the management of cancer. Lancet Oncol. 2003 Jun;4(6):351-8.

18 Brueckner B, Lyko F. DNA methyltransferase inhibitors: old and new drugs for an epigenetic cancer therapy. Trends Pharmacol Sci. 2004 Nov;25(11):551-4.

19 Karahoca M, Momparler RL. Pharmacokinetic and pharmacodynamic analysis of 5-aza- $2^{\prime}$ deoxycytidine (decitabine) in the design of its dose-schedule for cancer therapy. Clin Epigenetics. 2013 Feb;5(1):3.
20 Lübbert M, Rüter BH, Claus R, Schmoor C, Schmid M, Germing U, et al. A multicenter phase II trial of decitabine as first-line treatment for older patients with acute myeloid leukemia judged unfit for induction chemotherapy. Haematologica. 2012 Mar;97(3): 393-401.

21 Issa JP, Garcia-Manero G, Giles FJ, Mannari R, Thomas D, Faderl S, et al. Phase 1 study of low-dose prolonged exposure schedules of the hypomethylating agent 5-aza-2' deoxycytidine (decitabine) in hematopoietic malignancies. Blood. 2004 Mar;103(5):163540.

22 Lal G, Bromberg JS. Epigenetic mechanisms of regulation of Foxp3 expression. Blood. 2009 Oct;114(18):3727-35.

23 Katoh H, Zheng P, Liu Y. FOXP3: genetic and epigenetic implications for autoimmunity. J Autoimmun. 2013 Mar;41:72-8.

24 Moon C, Kim SH, Park KS, Choi BK, Lee HS, Park JB, et al. Use of epigenetic modification to induce FOXP3 expression in naïve T cells. Transplant Proc. 2009 Jun;41(5):1848-54.

25 Lal G, Zhang N, van der Touw W, Ding Y, Ju W, Bottinger EP, et al. Epigenetic regulation of Foxp3 expression in regulatory $\mathrm{T}$ cells by DNA methylation. J Immunol. 2009 Jan; 182(1):259-73

26 Polansky JK, Kretschmer K, Freyer J, Floess S, Garbe A, Baron U, et al. DNA methylation controls Foxp3 gene expression. Eur J Immunol. 2008 Jun;38(6):1654-63. 
27 Zheng Q, Xu Y, Liu Y, Zhang B, Li X, Guo F, et al. Induction of Foxp3 demethylation increases regulatory CD4+CD25+ T cells and prevents the occurrence of diabetes in mice. J Mol Med (Berl). 2009 Dec;87(12):1191-205.

28 Sánchez-Abarca LI, Gutierrez-Cosio S, Santamaría C, Caballero-Velazquez T, Blanco B, Herrero-Sánchez $\mathrm{C}$, et al. Immunomodulatory effect of 5-azacytidine (5-azaC): potential role in the transplantation setting. Blood. 2010 Jan;115(1):107-21.

29 Kanamori M, Nakatsukasa H, Okada M, Lu Q, Yoshimura A. Induced Regulatory T Cells: Their Development, Stability, and Applications. Trends Immunol. 2016 Nov;37(11): 803-11.

30 Luo X, Zhang Q, Liu V, Xia Z, Pothoven KL, Lee C. Cutting edge: TGF-beta-induced expression of Foxp3 in $\mathrm{T}$ cells is mediated through inactivation of ERK. J Immunol. 2008 Mar; 180(5):2757-61.

31 Kehrmann J, Tatura R, Zeschnigk M, ProbstKepper M, Geffers R, Steinmann J, et al. Impact of 5-aza- $2^{\prime}$-deoxycytidine and epigallocatechin-3-gallate for induction of human regulatory $\mathrm{T}$ cells. Immunology. $2014 \mathrm{Jul}$; 142(3):384-95.

32 Nakanishi M, Rosenberg DW. Multifaceted roles of PGE2 in inflammation and cancer. Semin Immunopathol. 2013 Mar;35(2):12337.

33 Uematsu S, Matsumoto M, Takeda K, Akira S. Lipopolysaccharide-dependent prostaglan$\operatorname{din} E(2)$ production is regulated by the glutathione-dependent prostaglandin $\mathrm{E}(2)$ synthase gene induced by the Toll-like receptor 4/MyD88/NF-IL6 pathway. J Immunol. 2002 Jun;168(11):5811-6.

34 Joshi PC, Zhou X, Cuchens M, Jones Q. Prostaglandin E2 suppressed IL-15-mediated human NK cell function through down-regulation of common gamma-chain. J Immunol. 2001 Jan;166(2):885-91.

35 Martinet L, Jean C, Dietrich G, Fournié JJ, Poupot R. PGE2 inhibits natural killer and gamma delta $\mathrm{T}$ cell cytotoxicity triggered by NKR and TCR through a cAMP-mediated PKA type I-dependent signaling. Biochem Pharmacol. 2010 Sep;80(6):838-45.

36 Boniface K, Bak-Jensen KS, Li Y, Blumenschein WM, McGeachy MJ, McClanahan TK, et al. Prostaglandin E2 regulates Th17 cell differentiation and function through cyclic AMP and EP2/EP4 receptor signaling. J Exp Med. 2009 Mar;206(3):535-48.

37 Kochel TJ, Goloubeva OG, Fulton AM. Upregulation of Cyclooxygenase-2/Prostaglandin E2 (COX-2/PGE2) Pathway Member Multiple Drug Resistance-Associated Protein 4 (MRP4) and Downregulation of Prostaglandin Transporter (PGT) and 15-Prostaglandin Dehydrogenase (15-PGDH) in Triple-Negative Breast Cancer. Breast Cancer (Auckl). 2016 May;10:61-70.
38 Starczewski M, Voigtmann R, Peskar BA, Peskar BM. Plasma levels of 15-keto-13,14-dihydro-prostaglandin E2 in patients with bronchogenic carcinoma. Prostaglandins Leukot Med. 1984 Mar;13(3):249-58.

39 Malachi T, Chaimoff C, Feller N, Halbrecht I. Prostaglandin E2 and cyclic AMP in tumor and plasma of breast cancer patients. J Cancer Res Clin Oncol. 1981;102(1):71-9.

40 Yuan XL, Chen L, Li MX, Dong P, Xue J, Wang J, et al. Elevated expression of Foxp3 in tumor-infiltrating Treg cells suppresses T-cell proliferation and contributes to gastric cancer progression in a COX-2-dependent manner. Clin Immunol. 2010 Mar;134(3):277-88.

41 Baratelli F, Lin Y, Zhu L, Yang SC, HeuzéVourc'h N, Zeng G, et al. Prostaglandin E2 induces FOXP3 gene expression and T regulatory cell function in human CD4+ T cells. J Immunol. 2005 Aug;175(3):1483-90.

42 Sharma S, Yang SC, Zhu L, Reckamp K, Gardner B, Baratelli F, et al. Tumor cyclooxygenase-2/prostaglandin E2-dependent promotion of FOXP3 expression and CD4+ CD25+ $\mathrm{T}$ regulatory cell activities in lung cancer. Cancer Res. 2005 Jun;65(12):5211-20.

43 Sinha P, Clements VK, Fulton AM, OstrandRosenberg S. Prostaglandin E2 promotes tumor progression by inducing myeloid-derived suppressor cells. Cancer Res. 2007 May; 67(9):4507-13.

44 Tomić S, Joksimović B, Bekić M, Vasiljević M, Milanović M, Čolić M, et al. Prostaglanin-E2 Potentiates the Suppressive Functions of $\mathrm{Hu}$ man Mononuclear Myeloid-Derived Suppressor Cells and Increases Their Capacity to Expand IL-10-Producing Regulatory T Cell Subsets. Front Immunol. 2019 Mar;10:475

45 de Vos D, van Overveld W. Decitabine: a historical review of the development of an epigenetic drug. Ann Hematol. 2005 Dec;84(S1 Suppl 1):3-8.

46 Jabbour E, Issa JP, Garcia-Manero G, Kantarjian $\mathrm{H}$. Evolution of decitabine development: accomplishments, ongoing investigations, and future strategies. Cancer. 2008 Jun; 112(11):2341-51.

47 Lemaire M, Chabot GG, Raynal NJ, Momparler LF, Hurtubise A, Bernstein ML, et al. Importance of dose-schedule of 5-aza- $2^{\prime}$ deoxycytidine for epigenetic therapy of cancer. BMC Cancer. 2008 May;8(1):128.

48 Gagnon J, Shaker S, Primeau M, Hurtubise A, Momparler RL. Interaction of 5-aza- $2^{\prime}$ deoxycytidine and depsipeptide on antineoplastic activity and activation of 14-3-3sigma, E-cadherin and tissue inhibitor of metalloproteinase 3 expression in human breast carcinoma cells. Anticancer Drugs. 2003 Mar; 14(3):193-202.

49 Egger G, Liang G, Aparicio A, Jones PA. Epigenetics in human disease and prospects for epigenetic therapy. Nature. 2004 May; 429(6990):457-63.

50 Saba HI. Decitabine in the treatment of myelodysplastic syndromes. Ther Clin Risk Manag. 2007 Oct;3(5):807-17.
51 Kim HP, Leonard WJ. CREB/ATF-dependent $\mathrm{T}$ cell receptor-induced FoxP3 gene expression: a role for DNA methylation. J Exp Med. 2007 Jul;204(7):1543-51.

52 Moore KW, de Waal Malefyt R, Coffman RL, O'Garra A. Interleukin-10 and the interleukin-10 receptor. Annu Rev Immunol. 2001; 19(1):683-765.

53 Tsuji-Takayama K, Suzuki M, Yamamoto M, Harashima A, Okochi A, Otani T, et al. The production of IL-10 by human regulatory T cells is enhanced by IL-2 through a STAT5responsive intronic enhancer in the IL-10 locus. J Immunol. 2008 Sep;181(6):3897-905.

54 Szalmás A, Bánáti F, Koroknai A, László B, Fehér E, Salamon D, et al. Lineage-specific silencing of human IL-10 gene expression by promoter methylation in cervical cancer cells. Eur J Cancer. 2008 May;44(7):1030-8.

55 Lee S, Margolin K. Cytokines in cancer immunotherapy. Cancers (Basel). 2011 Oct;3(4): 3856-93.

56 Lugade AA, Sorensen EW, Gerber SA, Moran JP, Frelinger JG, Lord EM. Radiation-induced IFN-gamma production within the tumor microenvironment influences antitumor immunity. J Immunol. 2008 Mar;180(5):3132-9.

57 Blankenstein T, Qin Z. The role of IFN-gamma in tumor transplantation immunity and inhibition of chemical carcinogenesis. Curr Opin Immunol. 2003 Apr;15(2):148-54.

58 Wang Z, Hong J, Sun W, Xu G, Li N, Chen X, et al. Role of IFN-gamma in induction of Foxp 3 and conversion of CD4+ CD25- T cells to CD4+ Tregs. J Clin Invest. 2006 Sep;116(9): 2434-41.

59 Yano S, Ghosh P, Kusaba H, Buchholz M, Longo DL. Effect of promoter methylation on the regulation of IFN-gamma gene during in vitro differentiation of human peripheral blood T cells into a Th2 population. J Immunol. 2003 Sep;171(5):2510-6.

60 Sahin M, Sahin E, Gümüslü S. Cyclooxygenase- 2 in cancer and angiogenesis. Angiology. 2009 Apr-May;60(2):242-53.

61 Chizzolini C, Brembilla NC. Prostaglandin E2: igniting the fire. Immunol Cell Biol. 2009 Oct;87(7):510-1.

62 Sahin E, Sahin M. Epigenetical Targeting of the FOXP3 Gene by S-Adenosylmethionine Diminishes the Suppressive Capacity of Regulatory T Cells Ex Vivo and Alters the Expression Profiles. J Immunother. 2019 Jan;42(1): 11-22.

63 Xia D, Wang D, Kim SH, Katoh H, DuBois RN. Prostaglandin E2 promotes intestinal tumor growth via DNA methylation. Nat Med. 2012 Jan;18(2):224-6.

64 Huang SK, Scruggs AM, Donaghy J, McEachin RC, Fisher AS, Richardson BC, et al. Prostaglandin $\mathrm{E}_{2}$ increases fibroblast gene-specific and global DNA methylation via increased DNA methyltransferase expression. FASEB J. 2012 Sep;26(9):3703-14. 